\title{
Qualidade de Serviços: Uma análise comparativa entre SERVQUAL E SERVPERF
}

\author{
Christiane de Melo Rêgo SOUTO \\ Universidad Complutense de Madri (UCM), Madrid, Espanha
}

\section{Jorge da Silva CORREIA-NETO}

Universidade Federal Rural de Pernambuco (UFRPE), Recife, PE, Brasil

\author{
Received 10 nov. 17; Accepted 5 dez. 17 \\ Evaluation System: Guest Article \\ Editor: Jose Lindenberg Julião Xavier Filho, Dr. \\ ISSN: $2594-8040$
}

To cite this paper: Souto, C. M. R., \& Correia-Neto, J. S. (2017). Qualidade de Serviços: Uma análise comparativa entre SERVQUAL e SERVPERF. Journal of Perspectives in Management-JPM, 1(1), p. 63-73.

\section{Resumo}

Desde os anos 1980 discute-se como mensurar a qualidade dos serviços prestados, especialmente por que cada vez mais tem-se algum tipo de serviço associado aos produtos comercializados. Entretanto, a escala proposta por Parasuraman, Zeithaml e Berry, nomeada SERVQUAL, não é uma unanimidade até hoje. Além das críticas quanto a diversos aspectos, inclusive estatísticos, a escala SERVPERF obtém resultados iguais ou melhores do que sua antecessora, mas mesmo assim também não é uma unanimidade. Em síntese, a SERVQUAL apresenta 22 variáveis dispostas nas seguintes dimensões: tangíveis, confiabilidade, receptividade, segurança e empatia. A SERVPERF utiliza as mesmas variáveis e dimensões, contudo, ao invés de analisar o gap existente entre as expectativas e a performance do serviço executado, capta a percepção do cliente apenas após o consumo do serviço. $\mathrm{O}$ presente ensaio, a partir da literatura da área de marketing de serviços, buscou apontar as vantagens e desvantagens de cada uma dessas escalas e assim propor à comunidade científica um novo debate, mais fundamentado, sobre as referidas escalas. $\mathrm{O}$ estudo demonstrou o crescimento do interesse no tema e como contribuição apontou as principais vantagens e desvantagens de cada uma dessas escalas.

Palavras-Chave: Qualidade de Serviços, SERVQUAL, SERVPERF. 


\section{Introdução}

Há mais de três décadas pesquisas sobre qualidade em serviços envolvem pesquisadores de Marketing, desde os trabalhos pioneiros de Holbrook e Corfman (1985), Parasuraman, Zeithaml e Berry (1985; 1989; 1991), Bitner (1990) e Cronin e Taylor (1992; 1994), entre outros. Atualmente é lugar comum reconhecer que um serviço de qualidade é de fundamental importância para se alcançar os objetivos estratégicos das empresas, por isso vem se tornando cada vez mais importante o monitoramento da qualidade de serviço como forma de avaliar o desempenho global das organizações, quer visem lucro ou não.

Os mais relevantes e seminais estudos sobre qualidade em serviços envolvem principalmente as publicações de Parasuraman, Zeithaml e Berry (1985; 1989; 1991), que ao proporem a escala SERVQUAL se basearam no paradigma da desconfirmação das expectativas, ou seja, na diferença medida entre o desempenho (entregue ao consumidor) e as expectativas que o consumidor tinha antes da experiência de consumo.

Por outro lado, os trabalhos de Cronin e Taylor (1992; 1994) argumentam que qualidade e satisfação são construtos diferentes e que a satisfação age como mediador, alterando as expectativas e avaliações sobre determinado serviço, por isso criticam o uso do paradigma da desconfirmação. A partir desse debate estes autores propõem a escala SERVPERF, que utiliza as mesmas variáveis da SERVQUAL, mas avalia apenas a experiência de consumo, deixando de lado a questão das expectativas.

Como ainda não existe um consenso acerca da melhor forma se operacionalizar a medição do construto qualidade percebida (Abreu \& Andrade, 2017), justifica-se um aprofundamento maior na análise das vantagens e desvantagens entre os principais modelos e escalas que operacionalizam essa medição, em especial, a SERVQUAL e a SERVPERF, sendo esse o problema central de pesquisa. Assim, este ensaio tem como objetivo investigar os conceitos de qualidade em serviços como um construto multidimensional que relaciona elementos como qualidade percebida, satisfação do cliente e atitude, com base em algumas das mais relevantes obras da literatura de marketing de serviços.

Para viabilizar esta análise, os autores (1) apresentam de forma sucinta cada um destes modelos e escalas, (2) as principais críticas que lhes são feitas e, por fim, (3) conclusões e questionamentos.

\section{Revisão da Literatura}

As propostas teóricas de alguns dos principais autores em marketing de serviços que analisaram profundamente o construto qualidade em serviço servirão se subsídio para o este trabalho. Desta forma será feita uma revisão histórica dos principais autores e questionadores deste construto para a futura análise do artigo. Além disso, também subsidiará esse estudo o trabalho de Mondo e Fiates (2017), que identificou 36 modelos e 211 indicadores de qualidade em serviços.

Nas últimas décadas vem crescendo a importância do setor de serviços em todas as economias mais desenvolvidas do mundo. Em mercados mais maduros como o bancário e o de aviação civil, entre outros, a concorrência tem feito com que as empresas busquem maiores índices de satisfação de seus clientes como forma de ampliação de seus mercados e de retenção de clientes, já que maiores níveis de satisfação levam a um maior incremento de participação de mercado por unidade de tempo (Pascale \& Simon, 1997; Babich, 2001; Lovelock \& Wright, 2001). Como satisfação e qualidade estão intimamente ligados, serão conceituados a seguir.

Durante os anos 1980 o foco das pesquisas sobre o marketing de serviços esteve situado no construto da qualidade, que seria uma avaliação global e de longo prazo que o consumidor faz sobre um determinado serviço, algo semelhante à atitude (Bateson \& Hoffman, 2001; Parasuraman, Zeithaml \& Berry, 1988; 1991). Essas semelhanças existem, pois as atitudes são tratadas como um conceito global vinculado às predisposições individuais (Holbrook \& Corfman, 1985). Solomon (1998, p. 277), em concordância com estes, define atitude como uma "predisposição em avaliar um objetivo ou produto positiva ou negativamente". Dadas as características únicas dos serviços (intangibilidade, heterogeneidade e inseparabilidade entre produção e consumo) (Zeithaml \& Bitner, 2003), a qualidade pode ser vista também como uma comparação entre o serviço recebido e os níveis de serviço desejado e adequado.

Se a qualidade percebida estiver entre esses níveis (zona de tolerância), os consumidores avaliarão o serviço como sendo de qualidade (Parasuraman, Zeithaml \& Berry, 1988; 1991). Para esse ensaio, qualidade é a medida de quanto um serviço atendeu às expectativas do consumidor, numa base consistente (Lewis \& Booms, 1983).

Durante os anos 1990 a produção acadêmica (a exemplo dos trabalhos de Swan e Oliver (1991), Rust e Zahorik (1993) e Oliver (1996)), focou a 
satisfação dos clientes. Satisfação é algo mais dinâmico que a qualidade, posto que é uma avaliação de uma transação (encontro) de serviço específica, recém experienciada (Bateson \& Hoffman, 2001). Além disso, é a resultante de diversos elementos, tais como (Farias \& Santos, 1998):

- Expectativas: Probabilidade de ocorrência;

- Performance: Resultado geral da execução do serviço;

- Desconfirmação: Diferencial entre a performance e as expectativas do consumidor;

- Esforço empreendido pelo consumidor para adquirir/contratar aquele serviço;

- Controle: O quanto aquele serviço pode ser gerido pelo consumidor;

- Atribuições: A quem o cliente atribuirá sua (in) satisfação;

- Equidade: Sentimento de justiça e equilíbrio na relação de troca;

- Emoções: Sentimentos.

Os resultados das pesquisas de marketing feitas nas décadas de 1980 e 1990 culminaram com alguns modelos de mensuração da qualidade de serviços. Os dois modelos mais difundidos entre os pesquisadores utilizaram as escalas SERVQUAL (1985) e SERVPERF (1992), que serão apresentadas a seguir.

\section{Modelo SERVQUAL}

Os artigos publicados por Parasuraman, Zeithaml e Berry em 1985 e 1988 (revisados em 1991), podem ser considerados como seminais na área de qualidade em serviços. Eles desenvolveram uma escala genérica para medir a qualidade de serviços em qualquer segmento econômico (acrescentando novas variáveis ao modelo básico).

Os autores fizeram uma pesquisa exploratória utilizando focus groups (com usuários de serviços bancários, de reparos de automóveis, seguros e cartões de crédito) e entrevistas em profundidade (com executivos de empresas destes ramos). Dessa pesquisa emergiu o Modelo da Qualidade em Serviços, mais conhecido como Modelo das Lacunas, pois ele identifica cinco grandes lacunas de percepção (Bateson \& Hoffman, 2001; Lovelock \& Wrigth, 2001):

- Diferença entre o que os consumidores esperam de um serviço e o que a gerência percebe que os consumidores esperam;
- Diferença entre o que a gerência percebe que os consumidores esperam e as especificações de qualidade determinadas para execução do serviço;

- Diferença entre as especificações de qualidade determinadas para a execução do serviço e a verdadeira qualidade da execução do serviço;

- Diferença entre a verdadeira qualidade da execução do serviço e a qualidade da execução do serviço descrita nas comunicações externa da empresa e;

- Diferença entre a qualidade percebida e a qualidade esperada pelo consumidor. Nesse estudo foram identificadas dez dimensões que determinariam a qualidade dos serviços.

Em 1988, com base nos estudos de 1985, Parasuraman, Zeithaml e Berry publicaram a SERVQUAL, que até hoje continua sendo a mais utilizada escala para medição da qualidade de serviços (Abreu \& Andrade, 2017). Utilizando diversos elementos estatísticos (análise fatorial, correlação, alpha de Cronbach, e ANOVA, entre outros), realizaram a purificação e condensação das 97 (noventa e sete) variáveis diagnosticadas durante a pesquisa de 1985 e implementaram a SERVQUAL com 22 (vinte e duas) variáveis, aglutinadas em cinco dimensões (tangíveis, confiabilidade, receptividade, segurança e empatia) que efetivamente medem o construto qualidade em serviços, como apresentado na Figura 1. Essas 22 (vinte e duas) variáveis devem ser pesquisadas tanto para as expectativas como para a performance dos serviços avaliados pelos consumidores, para que possa ser feito o diferencial entre elas.

Um resultado potencial da aplicação da SERVQUAL é a determinação da importância relativa que os consumidores de uma determinada empresa ou segmento dão a cada dimensão, viabilizando uma melhor alocação de investimentos no sentido de melhorar a qualidade percebida pelos mesmos. Com a realização periódica dessas pesquisas, consegue-se acompanhar a performance geral da empresa (naqueles atributos específicos) junto a seus consumidores.

Mas a utilização do esquema Performance menos Expectativa incomodava pesquisadores como Cronin e Taylor que, baseados na SERVQUAL, elaboram a escala SERVPERF. 


\section{FIGURA 1}

Qualidade de serviço conforme è conceituada por Parasuraman, Zeithaml e Berry (1988)

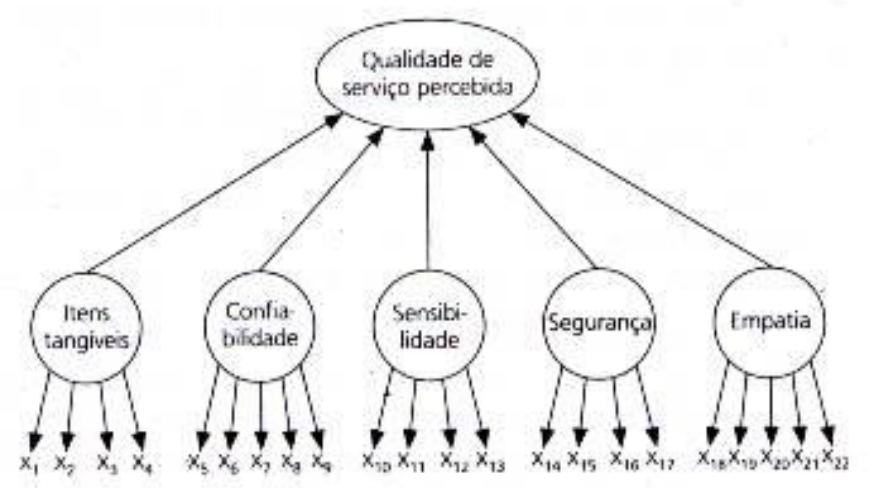

Fonte: Adaptado de Parasuraman, Berry e Zeithaml (1988).

\section{Modelo SERVPERF}

A conceituação e as medidas da qualidade de serviço são também investigadas por Cronin e Taylor, em 1992. Os autores criaram um modelo de mensuração da qualidade de serviços chamado SERVPERF, que é idêntico à escala SERVQUAL, porém sem o conjunto de questões referentes às expectativas (se concentra apenas na performance já executada). Dessa forma, utiliza apenas os 22 (vinte e dois) itens de desempenho, com uma escala Likert de 5 ou 7 pontos e tem nos extremos "Discordo fortemente" e "Concordo fortemente".

Como qualidade de serviço é um construto abstrato e ilusório, de difícil mensuração, Cronin e Taylor (1992) acrescentaram, a esses itens de desempenho, questões para analisar as relações entre a qualidade de serviço, satisfação do consumidor e intenções de compra (chamadas medidas autorelatadas).

Corroborando com esses questionamentos acerca da mensuração das expectativas, Carman (1990) afirma que as expectativas mudam de acordo com a familiaridade que o consumidor tem com aquele serviço e com experiências anteriores. Além disso, quando as expectativas eram medidas após a execução do serviço, junto com a performance, recebiam uma forte influência da desta.

Muitos autores como Brown, Churchill Jr., Gilbert e Peter (1993), acreditam que exista uma linha tênue entre satisfação e atitude na definição da qualidade de serviço relacionalmente estabelecida por Parasuraman, Zeithaml e Berry em 1985.
Segundo Cronin e Taylor (1992) a qualidade percebida conduzindo a um alto nível de satisfação do consumidor, visão defendida por Parasuraman, Zeithaml e Berry (1985; 1988), é uma visão equivocada, pois evidências posteriores parecem sugerir que a satisfação é, na verdade, um antecedente da qualidade de serviço.

Assim, Cronin e Taylor (1992) avaliam (1) um método alternativo que se baseia unicamente na performance do serviço e, (2) a ordem da relação causal entre qualidade de serviço e satisfação de consumidor, além do impacto da qualidade de serviço e satisfação do consumidor nas intenções de compra.

Com base numa pesquisa realizada com 600 respondentes durante o verão de 1988, foram reunidas respostas sobre a qualidade de serviço oferecida por duas empresas de cada um dos quatro setores escolhidos: bancos, controles de pragas, tinturarias e fast-food. Além das perguntas utilizadas pela SERVQUAL foram adicionadas ao questionário as seguintes medidas auto-relatadas de satisfação do consumidor e intenções de compra:

- No próximo ano, meu uso de XYZ será... (para avaliação de comportamento de compras futuras);

- A qualidade dos serviços de XYZ é... (para avaliar a qualidade global) e;

- Meus sentimentos em relação aos serviços de XYZ podem ser melhor descritos como... (para avaliar satisfação). 
As principais conclusões encontradas pelos autores foram: (1) uma medida de qualidade baseada na performance (SERVPERF) pode ser a melhor para se medir a qualidade de serviço; (2) as atuais conceituações e avaliações de marketing sobre qualidade de serviço são baseadas em um paradigma falho porque fundamentam-se no paradigma da satisfação e não em um modelo de atitude e a análise empírica dos modelos estruturais sugeriu que o modelo se confirmou em apenas dois dos quatro setores estudados.

Examinando a dimensionalidade da escala SERVQUAL por meio de análise fatorial, os autores não confirmaram a existência de cinco dimensões propostas por Parasuraman, Zeithaml e Berry (1988) em nenhuma das amostras da pesquisa feita, diminuindo sua generalidade. Além disso, comparando SERVQUAL com SERVPERF concluem que a escala SERVPERF explica melhor a variação da medida global de qualidade de serviço. Assim os autores sugerem que as medidas propostas baseadas em desempenho forneçam uma exemplificação de qualidade de serviço mais válida com relação ao construto devido à validade do conteúdo.
Vale ainda salientar que, segundo Peters Filho (2002), o modelo SERVQUAL foi aprimorado pelo modelo SERVPERF por sintetizar em uma só medida - a do desempenho - a satisfação do consumidor com o serviço, em vez da desconfirmação de expectativas e desempenho.

Para analisar a credibilidade das escalas SERVQUAL e SERVPERF, Cronin e Taylor (1992) utilizaram-se dos modelos estruturais, onde confirmaram algumas hipóteses quanto à relação causal entre as variáveis satisfação, qualidade e intenção de compra.

\section{Modelos Estruturais}

Buscando os nexos de causalidade entre a qualidade de serviço, satisfação do cliente e intenções de compra, Cronin e Taylor (1992) utilizaram os modelos estruturais, avaliando simultaneamente as escalas SERVQUAL e SERVPERF (Figura 2).

FIGURA 2

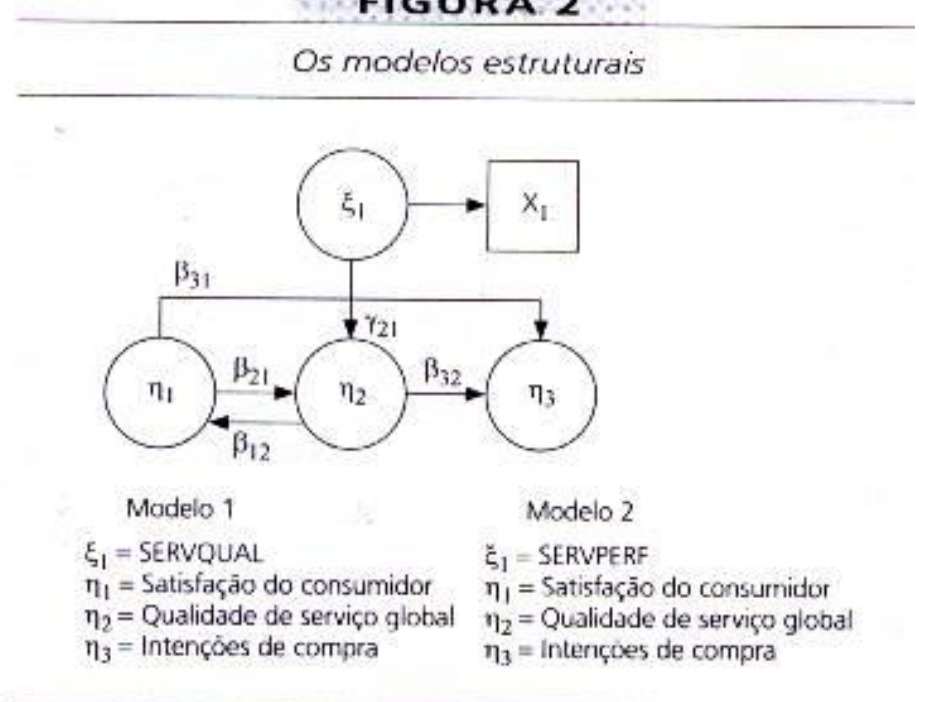

Fonte: Cronin e Taylor (1992).

Os modelos estruturais são usados para estender a consideração das escalas, assim como para considerar as três questões restantes de pesquisa (1) a satisfação do cliente é um antecedente da qualidade de serviço percebida, (2) a satisfação do cliente tem um impacto significativo nas intenções de compra, (3) a qualidade de serviço percebida tem um impacto significativo nas intenções de compra. Nessa avaliação ficou claro que os modelos 1 e 2 são idênticos (a única diferença entre os dois modelos é a medida usada para a qualidade de serviço).

A escala SERVPERF teve um excelente ajuste nos quatro setores avaliados enquanto que a SERVQUAL só teve um bom ajuste em dois dos quatro setores (bancos e fast-food). Devido à sua superioridade, foi utilizada para avaliar se: (1) a satisfação do cliente é um antecedente da qualidade 
de serviço percebida $\beta 21 / \beta 12$; (2) a satisfação do consumidor tem um impacto significativo nas intenções de compra $\beta 31$; (3) a qualidade de serviço percebida tem um impacto significativo nas intenções de compra $\beta 32$ (Cronin \& Taylor 1992).

A ordem causal do relacionamento entre qualidade de serviço e satisfação é apontada atualmente como sendo a satisfação antecedendo a qualidade de serviço (Bitner, 1990). No entanto as pesquisas indicam que o conceito de qualidade de serviço percebida realmente leva à satisfação como atestam Parasuraman, Zeithaml e Berry (1985; 1988). Nessa mesma linha, verificou-se no estudo de Cronin e Taylor (1992) que a qualidade teve um efeito significativo na satisfação do consumidor, nas quatro amostras pesquisadas, sendo esta também a posição dos autores deste artigo.

Quanto aos efeitos da qualidade de serviço e satisfação em relação às intenções de compra, observou-se que a satisfação parece ter um efeito mais forte e mais consistente nas intenções de compra que a qualidade de serviço ( $\beta 31$ ). A qualidade de serviço não teve um impacto significativo nas intenções de compra em nenhuma das amostras ( $\beta 32)$ (CRONIN; TAYLOR, 1992).

\section{Críticas aos Modelos de Mensuração da Qualidade em Serviços}

\subsection{SERVQUAL}

Estudos realizados por inúmeros autores, por exemplo Carman (1990), Finn e Lamb (1991), Babakus e Boller (1992), Cronin e Taylor (1992; 1994) e Brown et al. (1993) questionaram a operacionalização do modelo SERVQUAL, alegando que a fórmula "Percepção - Expectativa" $(\mathrm{P}-\mathrm{E})$, não seria a ideal para mensurar qualidade de serviço. Eles alegam que as expectativas podem já estar presentes nas percepções dos respondentes, pois estas sozinhas já podem ser uma avaliação global de qualidade (DUTRA, 2001).

Apesar do refinamento da SERVQUAL realizado por Parasuraman, Zeithaml e Berry (1991), alguns estudiosos seguem dizendo que existem falhas e que há a necessidade de promover pesquisas sobre sua confiabilidade. Por exemplo, Lam e Woo (1997) testaram a aplicabilidade e confiabilidade da escala longitudinalmente. Eles usaram o método de teste reteste a curto e longo prazo. Os resultados mostraram que o modelo SERVQUAL não é estável ao longo do tempo e apresentou uma correlação insignificante entre os resultados do teste e do [re]teste.

Mesmo sendo uma das medidas mais populares a SERVQUAL tem problemas sérios na conceituação de qualidade de serviço como um resultado diferencial, segundo Brown et al. (1993). Estes autores confirmaram através de pesquisas empíricas que a SERVQUAL tem um bom desempenho, mas que as expectativas possuem poucas correlações com medidas de outros teoricamente relacionados, confirmando as conclusões de Cronin e Taylor (1992). Outro problema encontrado por Brown et al. (1993) tem relação com a dimensionalidade da escala SERVQUAL, pois em seus estudos ela não se repetiu, representando um construto unidimensional, e não um construto com cinco dimensões como proposto por Parasuraman, Zeithaml e Berry (1988). Os autores sugerem que seja utilizada uma escala de resultado não-diferencial, mais parecida com o SERVPERF.

Marchetti, Prado e Silva (1998) identificaram outros problemas operacionais da SERVQUAL, como a questão de tempo de aplicação dos questionários que é muito longo, dado que se aplica a escala duas vezes (uma para medir as expectativas e outra para mensurar a performance), o que torna a escala complexa e a aplicação mais cansativa para os respondentes. No entanto o autor não questiona a utilização da referida escala.

\subsection{SERVPERF}

Avaliar apenas a performance pode levar a distorções, principalmente em mercados multifacetados (como em termos sociais, culturais e econômicos, entre outros). Segundo Marchetti, Prado e Silva (1998) com a mensuração apenas da performance como indicador da qualidade de serviço, perde-se a referência das expectativas, que podem variar de região para região e de segmento para segmento, o que contribui para a não parcimônia do modelo.

Mas as críticas mais embasadas vêm dos próprios autores da SERVQUAL, que em 1994 publicaram um artigo refutando as críticas feitas pelos pesquisadores Cronin e Taylor (1992) e Teas (1993). Essas críticas foram divididas em questões conceituais, metodológicas / analíticas e práticas.

Com relação às criticas conceituais feitas por Cronin e Taylor (C\&T) (1992): 
- Falta de fundamentação teórica ou prática para embasar a ideia das lacunas entre expectativas e performance na literatura de qualidade de serviços: Parasuraman, Zeithaml e Berry apresentam 7 pesquisas de autores renomados, inclusive uma que é citada pelos próprios C\&T;

- Formação da atitude $x$ medição da atitude: Parasuraman, Zeithaml e Berry afirmam que a SERVQUAL não está preocupada com a formação da atitude, mas na sua medição num dado ponto no tempo;

- Link entre qualidade e satisfação: Parasuraman, Zeithaml e Berry afirmam que agora concordam com a maioria dos estudiosos no assunto (citados nominalmente) que a qualidade dos serviços conduz à satisfação dos consumidores (ponto que não estava tão claro para eles na época);

- Inter-relacionamento entre as 5 dimensões: Parasuraman, Zeithaml e Berry assumem esse inter-relacionamento, evidenciado pela necessidade do uso de rotações oblíquas de fatores de solução, mas não aceitam a unidimensionalidade;

- Variância: apenas 1 item da escala SERVQUAL fica com variância menor que $50 \%$, recomendado por Bagozzi e Yi (1988) como limite mínimo de um construto latente. Além disso, a variância nos 4 segmentos econômicos analisados era menor no SERVQUAL $(32,4 \%)$ que no SERVPERF $(42,6 \%)$;

- Análise de fator oblíquo: apesar de C\&T afirmarem que conduziram uma análise de fator oblíquo, seus relatórios não foram claros em demonstrar se essa carga foi feita com matrizes de carga rotacionada ou não rotacionada. Se ela foi pré-rotacionada, interpretá-las para inferir sobre a unidimensionalidade é questionável;

- SERVQUAL A SERVPERF como construtos unidimensionais por conta do alpha: Parasuraman, Zeithaml e Berry citam diversos autores que afirmam que o coeficiente alpha é uma estimativa de confiabilidade e não um necessário e suficiente indicativo de unidimensionalidade;

- Validade: SERVQUAL e SERVPERF se saem muito bem em todos os critérios de validade utilizados por C\&T. A validade convergente também é igual entre as duas (o que impediria C\&T de afirmar que a SERVPERF seria melhor). A validade discriminatória da SERVQUAL também seria maior que a SERVPERF porque a inter-correlação média dos construtos nas duas escalas é a mesma;

- Uso do modelo estrutural $\mathbf{x}$ unidimensionalidade: Parasuraman, Zeithaml e Berry criticam essa comparação afirmando primeiramente que não concordam que qualidade de serviços, satisfação do consumidor e intenção de compra sejam construtos unidimensionais e, depois, que as hipóteses 2, 3 e 4 só se relacionam com a parte inferior do modelo estrutural (figura 2);

- Escalas unidimensionais não dão liberdade para testes de correlação: o fato das correlações (qualidade do serviço $\mathrm{x}$ intenção de compra = .5334) e (satisfação do consumidor $x$ intenção de compra $=.5272$ ) serem praticamente idênticas, confirma a ideia de uma correlação direta entre qualidade e satisfação (diferentemente do que foi colocado por $\mathrm{C} \& \mathrm{~T}$ ) e;

- Ferramenta de diagnóstico: a SERVQUAL teria mais condições de apontar áreas deficientes na empresa, já que é mais detalhista (não apresenta o resultado pronto, a desconfirmação).

Os autores concordam com as críticas de Parasuraman, Zeithaml e Berry a C\&T, mas avaliam que de certa forma elas apenas reforçam a proximidade entre estas duas escalas.

Com relação às criticas conceituais feitas por Teas (1993):

- A resultante performance menos expectativa só é problemática sob certas condições e tipos de atributos: essa problemática só acontece com atributos vetoriais (aqueles que cujos pontos ideais para o consumidor estariam num nível infinito), como por exemplo, a camaradagem de um vendedor numa loja de varejo;

- Ponto ideal: para Teas (1993), o ponto ideal seria o nível aceitável de performance, sob circunstancias perfeitas. Para Parasuraman, Zeithaml e Berry ultrapassar esse ponto ideal seria inútil;

- Expectativa Essencial: são os atributos que os consumidores acreditam que sejam essenciais para que uma empresa tenha qualidade, sendo na realidade, um reforço para a necessidade de se medir as expectativas;

- Congruência entre as definições conceitual e operacional para medir expectativas: Parasuraman, Zeithaml e Berry não concordam com as aludidas dificuldades de interpretação das questões relativas a percepções e ainda criticam a utilização das questões abertas de Teas (1993);

- Uso de 8 modelos de qualidade em serviços: avaliando cada um dos modelos, Parasuraman, Zeithaml e Berry apresentam diversas críticas, inclusive contra as ideias dos pontos ideal e clássico. 
Os autores concordam com as críticas de Parasuraman, Zeithaml e Berry ao trabalho de Teas (1993), mas acreditam que as mesmas não tiveram tanta profundidade quanto as feitas ao trabalho de C\&T, já que o mesmo focou as suas críticas na problemática do ponto ideal.

\section{Conclusões}

Este ensaio teve como objetivo principal oferecer uma visão geral e crítica sobre os principais modelos de mensuração de qualidade de serviços, satisfação e intenção de compra (SERVQUAL e SERVPERF).

Nesse contexto, os autores avaliam que ainda existem muitas controvérsias sobre qual o melhor modelo para o momento atual. Analisando a literatura de marketing de serviços, até hoje estas divergências persistem, incluindo ainda outros modelos, como o da Desconfirmação (Marchetti, Prado \& Silva, 1998), modelos que carecem de melhor análise em estudos posteriores. Além disso, como o estudo bibliométrico de Abreu e Andrade (2017) aponta, há um crescente número de artigos sendo publicados que se utilizam ou discutem sobre essas escalas, especialmente a SERVQUAL, totalizando 120 artigos em periódicos (coletados a partir da Web of Science) só em 2016.

As principais vantagens e desvantagens dos modelos de mensuração da qualidade de serviços analisados pelos autores estão relacionados no Quadro 1.

\section{Quadro 1. Vantagens e desvantagens dos modelos SERVQUAL e SERVPERF}

\section{SERVQUAL}

\begin{tabular}{|c|c|c|}
\hline Vantagens & $\begin{array}{l}\text { - Modelo básico para todos os setores } \\
\text { empresariais } \\
\text { - Identificação dos pontos focais de qualidade } \\
\text { na percepção dos clientes } \\
\text { - Validade convergente e discriminatória }\end{array}$ & $\begin{array}{l}\text { - Modelo básico para todos os setores } \\
\text { empresariais } \\
\text { - Validade convergente e discriminatória } \\
\text { - Tempo de coleta menor } \\
\text { - Menor custo operacional }\end{array}$ \\
\hline Desvantagens & $\begin{array}{ll}\text { - } & \text { Instabilidade ao longo do tempo } \\
\text { - } & \text { Tempo de coleta maior }\end{array}$ & - Instabilidade ao longo do tempo \\
\hline
\end{tabular}

Fonte: Elaborado pelos Autores.

Os autores acreditam ser necessário um maior aprofundamento teórico e empírico para estabelecer um real modelo de facto para a mensuração destes construtos. No entanto, concordam que ambos os modelos são válidos, deste modo, tendo o SERVPERF consistência semelhante ao SERVQUAL e menor custo e tempo de operacionalização, apontam o SERVPERF como a ferramenta mais apropriada para o uso prático, mesmo que na pesquisa realizada (desk research), cerca de $90 \%$ dos artigos empíricos tenham se utilizado da SERVQUAL.

Ainda assim, os dois modelos continuam sendo referência ainda hoje, como apontam os estudos de Santos, Costa e Mondo (2014), que propuseram uma tipologia dos serviços públicos à luz do marketing de serviços, na proposta de Mondo e Fiates (2017) de um modelo específico de qualidade em serviços turísticos, no estudo de Ingaldi (2016) avaliando o serviço de transportes, e na análise bibliométrica acerca da SERVQUAL promovida por Abreu e Andrade (2017).

\section{Referências}

Abreu, A. A., \& Andrade, D. M. (2017). Tudo começa na SERVQUAL: análise bibliométrica sobre o tema qualidade em serviços. In: Seminários em Administração (SEMEAD), XX, São Paulo (SP), 8-10 Nov.

Angur, M., Nataraajan, R., \& Jahera, J. (1999). Service quality in the banking industry: an assessment in a developing economy. The International Journal of Bank Marketing Bradford.

Babakus, E., \& Mangold, W. G. (1992). An empirical assessment of the SERVQUAL scale. Journal of Business Research, 24.

Babich, P. (2001). Customer Satisfaction - How Good Is Good Enough? Quality Progress (December 1992, p. 65-67).

Bagozzi, R. R.; Yi, Y. (1988) On the Evaluation of Structural Equation Models. 1988. Journal of the Academy of Marketing Science, v. 16, n. 1, p. 744-94.

Bateson, J., \& Hoffman, K. (2001). Marketing de Serviços. 4. ed. Porto Alegre: Bookman. 
Bitner, M. J. (1990). Evaluating Service Encounter: The effects of physical surroundings and employee responses. Journal of Marketing, 54, p. 69-82.

Bitner, M. J., Boams, B.H., Tetreault, M. S. (1990). The Service Encounter: Diagnosing Favorable and Unfavorable Incidents. Journal of Marketing, 54 (1), p. 71-84.

Brown, T. J., Churchill, Jr., Gilbert A., \& Peter, J. Paul. (1993). Research note: improving the measurement of service quality. Journal of Retailing, 69 (1), spring.

Carman, J. M. (1990). Consumer perceptions of service quality: An assessment of the SERVQUAL dimensions. Journal of Retailing, 66 (1).

Cronin, J., \& Taylor, S. A. (1992). Measuring service quality: reexamination and extension. Journal of Marketing, 56 (3), July.

Cronin, J., \& Taylor, S. (1994). SERVPERF versus SERVQUAL. Journal of Marketing, 1, p. 12531.

Dutra, H. (2001). Percepção de Qualidade no Serviço Educacional: um estudo sobre o Curso de Administração da Faculdade de Ciências Humanas ESUDA. Dissertação... Dissertação (mestrado), Universidade Federal de Pernambuco.

Farias, S., \& Santos, R. (1998). Atributos de Satisfação nos Serviços de Hotelaria: uma perspectiva no segmento da $3^{\text {a }}$ idade. In: Encontro Anual da Associação Nacional de Pesquisa e Pós-Graduação em Administração (EnANPAD), Foz do Iguaçu (PR), 27-30 Set.

Finn, D. W., \& Jamb Jr., C. W. (1991). An evaluation of SERVQUAL scales in a retail setting. In: Holman, R. H. \& Solomon, M. R. (eds.). Advances in consumer research UT: Association for Consumer Research.

Holbrook, M. B., \& Corfman, K. P. (1985). Quality and value in the consumption experience: phaldrus rides again. Massachusetts: Lexington Books.

Ingaldi, M. K. (2016). Use of the SERVPERF method to evaluate service quality in the transport company. Independent Journal of Management \& Production (IJM\&P), 7(1), January/March.

Lam, S. S. K., \& Woo, K. S. (1997). Measuring service quality: a teste-retest reliability investigation of SERVQUAL. Journal of the Market Research Society, 19(2), April.

Lewis, Robert C., and Booms, Bernard H. (1983). The Marketing Aspects of Service Quality, Emerging Perspectives on Services Marketing. L. Berry, G. Shostack, and G. Upah, eds. American Marketing Association, Chicago, IL., pp. 99-107.
Llusar, J., \& Zornoza, C. (2000). Validity and reliability in perceived quality measurement models - An empirical investigation in Spanish ceramic companies. The International Journal of Quality \& Reliability Management Bradford.

Lovelock, C., \& Wright, L. (2001). Serviços: Marketing e Gestão. São Paulo: Saraiva.

Marchetti, R., Prado, P. H., \& Silva, A. R. (1998). Aspectos estratégicos e operacionais da avaliação da satisfação do usuário dos serviços públicos essenciais. In: Congresso Internacional Del CLAD, VI, Venezuela.

Matos, C. A., \& Veiga, R. T. (2000). Avaliação da qualidade percebida de serviços: um estudo em uma organização não-governamental. Caderno de Pesquisas em Administração, 7(3).

Mondo, T. S., \& Fiates, G. G. S. (2017). TOURQUAL: proposta de um protocolo para avaliação da qualidade dos serviços em atrativos turísticos. Brazilian Business Review, 14(4), p. 448-465.

Oliver, R. (1996). Customer Satisfaction Theory and Measurement. Journal of Marketing Management, Spring.

Parasuraman, A., Berry, L. L., \& Zeithaml, V. A. (1985). A conceptual model of service quality and its implications for future research. Journal of Marketing, 49.

Parasuraman, A., Berry, L. L., \& Zeithaml, V. A. (1988). SERVQUAL: A multiple-item scale for measuring consumer perceptions of service quality. Journal of Retailing, 64.

Parasuraman, A., Berry, L. L., \& Zeithaml, V. A. (1991). Refinement and reassessment of the SERVQUAL scale. Journal of Retailing, 67(4), winter.

Pascale, Q., \& Simon, R. (1997). Service marketing in the Australian advertising industry: a methodological study. The Journal of Services Marketing, 11(3), p.180-192.

Peters Filho, T. A. (2002). Proposição de modelagem da mensuração de valor percebido e envolvimento do consumidor em serviços. FECAP, 3(1), Janeiro.

Rust, R., \& Zahorik, A. (1993). Customer Satisfaction, Customer Retention, and Market Share. Journal of Retailing, 69(2), Summer.

Santos, A. R., Costa, J. I. P., \& Mondo, T. S. (2014). Tipologia dos serviços públicos à luz do marketing de serviços: uma proposição inicial. Revista Eletrônica Ciências da Administração e Turismo, 2(2), p. 15-32.

Solomon, M. R. (1998). Consumer behavior, bying, having e being. New Jersey: Prentice Hall.

Swan, J., \& Oliver, R. (1991). An Applied Analysis of Buyer Equity Perceptions and Satisfaction with Automobile Salespeople. Journal of 
Personal Selling \& Sales Management, 11(2), Spring.

Teas, R. K. (1993). Expectations, Performance

Evaluation, and Consumers' Perceptions of

Quality. Journal of Marketing, 57(4), 18-34.
Zeithaml, V., \& Bitner, M J. (2003). Marketing de serviços: a empresa com foco no cliente. Porto Alegre: Bookman.

\title{
Quality of Services: a comparative analysis between SERVQUAL and SERVPERF
}

\begin{abstract}
Since the 1980s it has been discussed how to measure the quality of the services provided, especially since more and more some type of service is associated with the products sold. However, the scale proposed by Parasuraman, Zeithaml and Berry, named SERVQUAL, is not unanimous until today. In addition to the criticisms on various aspects, including statistics, the SERVPERF scale achieves equal or better results than its predecessor, but it is not unanimous either. In summary, SERVQUAL presents 22 variables arranged in the following dimensions: tangible, reliability, receptivity, security and empathy. SERVPERF uses the same variables and dimensions, however, instead of analyzing the gap between the expectations and the performance of the service performed, it captures the perception of the customer only after consumption of the service. The present essay, from the service marketing literature, sought to point out the advantages and disadvantages of each of these scales and thus propose to the scientific community a new and more reasoned debate about the presented scales. The study demonstrated the growth of interest in the theme and as contribution pointed out the main advantages and disadvantages of each of these scales.
\end{abstract}

Keywords: Services Quality, SERVQUAL, SERVPERF.

\section{Calidad de servicio: un análisis comparativo entre SERVQUAL y SERVPERF}

\section{Resumen}

Desde los años 1980 se discute cómo medir la calidad de los servicios prestados, especialmente porque cada vez más se tiene algún tipo de servicio asociado a los productos comercializados. Sin embargo, la escala propuesta por Parasuraman, Zeithaml y Berry, nombrada SERVQUAL, no es una unanimidad hasta hoy. Además de las críticas sobre diversos aspectos, incluso estadísticos, la escala SERVPERF obtiene resultados iguales o mejores que su predecesora, pero aún así tampoco es una unanimidad. En síntesis, la SERVQUAL presenta 22 variables dispuestas en las siguientes dimensiones: tangibles, confiabilidad, receptividad, seguridad y empatía. La SERVPERF utiliza las mismas variables y dimensiones, sin embargo, en vez de analizar el gap existente entre las expectativas y el desempeño del servicio ejecutado, capta la percepción del cliente apenas después del consumo del servicio. El presente ensayo, a partir de la literatura del área de marketing de servicios, buscó apuntar las ventajas y desventajas de cada una de esas escalas y así proponer a la comunidad científica un nuevo debate, más fundamentado, sobre las referidas escalas. El estudio demostró el crecimiento del interés en el tema y como contribución apunta a las principales ventajas y desventajas de cada una de esas escalas.

Palabras Clave: Calidad de los Servicios, SERVQUAL, SERVPERF. 


\section{Sobre os Autores}

\section{Christiane de Melo Rêgo SOUTO}

Doutora em Administração pela Universidad Complutense de Madrid (UCM)

Av. Séneca, 2, 28040 Madrid, Espanha

E-mail: christiane.souto@gmail.com

\section{Jorge da Silva CORREIA-NETO}

Doutor em Administração pela Universidade Federal de Pernambuco (UFPE)

Rua Dom Manoel de Medeiros, S/N - Dois Irmãos - Recife - PE - Brasil - CEP 56.900-000.

E-mail: jorgecorreianeto@gmail.com 